\title{
Simultaneous Combination and Nearly Self-similar Pulse Compression of Five Pulses at Different Wavelengths
}

\author{
Jiayao Huang ${ }^{1}$, Feng Ye ${ }^{1}$, K. Nakkeeran ${ }^{2}$, P. K. A. Wai ${ }^{3}$ and Qian $\mathrm{Li}^{1, *}$ \\ ${ }^{I}$ School of Electronic and Computer Engineering, Peking University, Shenzhen 518055, China \\ ${ }^{2}$ School of Engineering, Fraser Noble Building, King's College, University of Aberdeen, Aberdeen, AB24 $3 U E$, U.K. \\ ${ }^{3}$ Photonics Research Centre, Department of Electronic and Information Engineering, The Hong Kong Polytechnic University, Hong Kong, \\ China. \\ *liqian@pkusz.edu.cn
}

\begin{abstract}
Numerical simulation of five raised-cosine pulses with spectral separation of $0.1 \mathrm{~nm}$ with each other merged into a single compressed pulse ( 12.8 compression factor) of nearly $50 \%$ of total input energy in a dispersion decreasing nonlinear fiber is reported. (C) 2021 The Author(s)
\end{abstract}

\section{Introduction}

Ultrashort pulses have found applications in frequency comb spectroscopy [1] and nonlinear optical imaging [2]. As an alternative scheme to produce ultrashort pulses without using complicated laser source(s), self-similar pulse compression has drawn vast scientific attention as it exploited the evolution of pulse(s) with a linear chirp in dispersion decreasing nonlinear fiber to achieve an efficient pulse compression. This kind of self-similar pulse compression maintained the advantages of integrally pedestal-free and nearly transform-limited features and pulse breakup as well avoided even at high peak powers during propagation [3]. Multiple raised-cosine (RC) optical pulses with the same central wavelengths can be combined and compressed in a dispersion decreasing nonlinear fiber to generate a single ultrashort pulse, which reveals that the final compressed pulse was a high-energy ultrashort pulse that contained major portion of the input light signal energy [4]. However, simultaneous combination and compression of multiple different-wavelength pulses is complex challenging dynamics that has never been reported. In this paper, we numerically demonstrate that five different-wavelength $\mathrm{RC}$ optical pulses coalesce into a single pulse and then evolve into an efficient self-similar compressed pulse in an exponentially decreasing dispersion nonlinear fiber, where the final compressed pulse reaches around 50\% energy of the total input pulses.

\section{Five Pulses Combination and Compression}

Five different-wavelength RC pulses expressed as follows are considered as the input light signal:

$$
\frac{\sqrt{P_{0}}}{2}\left\{1+\cos \left[\pi\left(t / T_{0}+1\right)\right]\right\} \exp (i 2 \pi \Delta v t) \exp \left(i \alpha_{20} t^{2} / 2\right), t / T_{0} \in[-5,5],
$$

where $T_{0}$ and $P_{0}$ account for the initial pulse width and initial peak power $\left(P_{0}=0.5\left|\beta_{20}\right| / \gamma / T_{0}^{2}\right)$, respectively. Simulation of the pulses propagation in dispersion decreasing nonlinear fiber is performed using generalized nonlinear Schrödinger equation (GNLSE) model [5]. In the GNLSE model, $\beta_{2}(\mathrm{z})$ decreases exponentially as $\beta_{2}(\mathrm{z})$ $=\beta_{20} \exp (-\sigma \mathrm{z})$, where $\beta_{20}$ is the group-velocity dispersion (GVD) at the beginning of the fiber $\mathrm{z}=0$. GVD decay rate $\sigma=\alpha_{20} \beta_{20}$, where $\alpha_{20}$ is the initial chirp and $\beta_{20}$ is the initial GVD. $\gamma$ and $\gamma_{1}$ are the nonlinearity coefficients. It is assumed that $\beta_{3}$ and $\gamma$ are constant in the fiber. $\gamma_{1} \approx \gamma / \omega_{0}$, where $\omega_{0}$ is the center frequency. The nonlinear response function is considered as $R(t)=\left(1-f_{\mathrm{R}}\right) \delta(t)+f_{\mathrm{R}} h_{\mathrm{R}}(t)$, where $f_{\mathrm{R}}=0.18$, and the Raman response function $h_{\mathrm{R}}$ is obtained by $h_{\mathrm{R}}(t)=\left(\tau_{1}^{2}+\tau_{2}^{2}\right) / \tau_{1} \tau_{2}^{2} \exp \left(-t / \tau_{2}\right) \sin \left(t / \tau_{1}\right)$, where $\tau_{1}=12.2 \mathrm{fs}$ and $\tau_{2}=32 \mathrm{fs}$. The pulse width $T_{0}$ is $1.374 \mathrm{ps}$ with a full width at half-maximum (FWHM) of 1 ps. The other fiber parameters are considered as $P_{0}=0.662 \mathrm{~W}, \alpha_{20}=-0.55$ $\mathrm{THz}^{2}, \beta_{20}=-25 \mathrm{ps}^{2} / \mathrm{km}, \beta_{3}=0.01 \mathrm{ps}^{3} / \mathrm{km}, \sigma=13.8 / \mathrm{km}, \gamma=10 / \mathrm{W} / \mathrm{km}$. The length of fiber $L$ is $300 \mathrm{~m} . \Delta v$ is the frequency spacing between any two adjacent pulse of the five input pulses that share the same initial chirp.

The central wavelengths of the input $5 \mathrm{RC}$ pulses are set as 1550,1550.1, 1550.2, 1550.3 and $1550.4 \mathrm{~nm}$ that corresponds to a frequency spacing of $\Delta v=12.5 \mathrm{GHz}$. As shown in Fig. 1(a), due to the influence of the shared chirp, five RC pulses are combined as a single pulse after propagating $246 \mathrm{~m}$ in the nonlinear fiber. During the remaining propagation, the combined pulse gets temporal compression to become a high energy output pulse. In Fig. 1(b), the compressed pulse spectrum depicts broadening and remains almost symmetric. Figure 1(c) reveals the pulse shapes at different fiber lengths. During the combination process, the energy gradually migrates towards the center pulse. The combination length is defined as a distance that the peak power of the pedestal of the combined pulse remains lower than $10 \%$ of the main peak. Figure 1(d) demonstrates that the combination length is $246 \mathrm{~m}$ at which the combined pulse FWHM is calculated as 0.206 ps. This illustrates that the pulse experiences a slight compression during the combination process. Numerically evaluated output compressed pulse and its best curve 
fitted hyperbolic secant profiled pulse are illustrated in Fig. 1(e), which represent that the peak power of the compressed pulse is 27.63 times as compared to any peak of the initial $5 \mathrm{RC}$ pulses (1). The FWHM is reduced from $1 \mathrm{ps}$ to $78.17 \mathrm{fs}$ with a compression factor of 12.8 . Note that the energy of the final compressed pulse is $1.70 \mathrm{pJ}$, which is around $50 \%$ of the total energy of the input light signal. Third-order dispersion generates small fluctuations near the trailing edge of the combined pulse causing little asymmetry in the final compressed pulse shape, but almost close to the fitted hyperbolic secant pulse. Figure 1(f) indicates that the compression factor of the output pulse is 12.8 and it nearly 10.2 value that is calculated from self-similar pulse compression analytical expression [3].

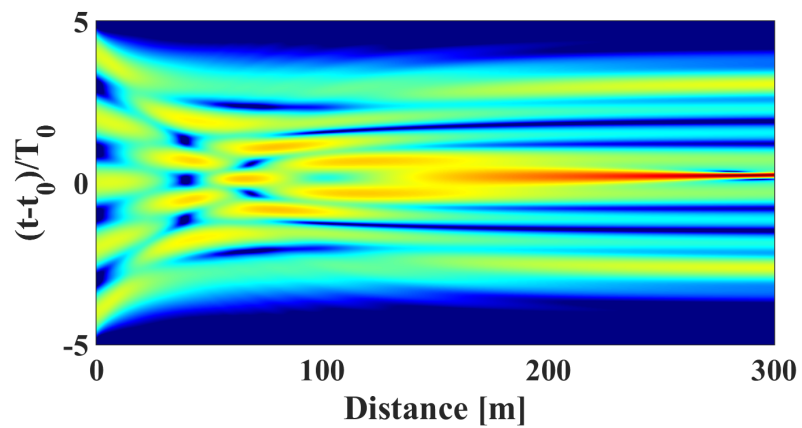

(a)

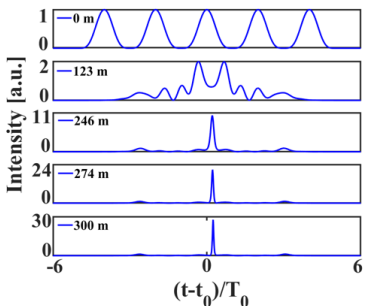

(c)

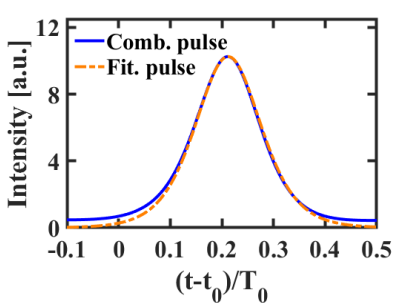

(d)

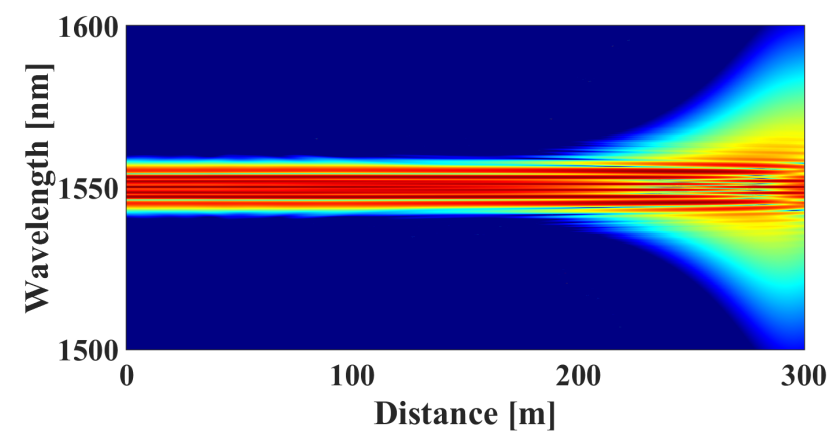

(b)

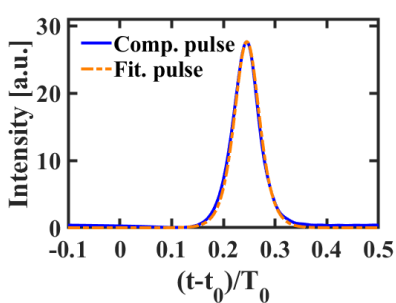

(e)

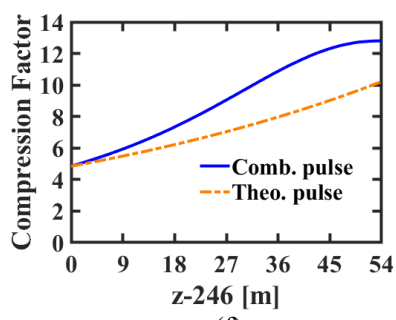

(f)

Fig. 1. Contour plot of (a) temporal and (b) spectral evolutions of the five chirped RC pulses during propagation in an exponentially dispersion decreasing nonlinear fiber. (c) Pulse shapes at $0 \mathrm{~m}$ (top), $123 \mathrm{~m}, 246 \mathrm{~m}, 274 \mathrm{~m}$ and $300 \mathrm{~m}$ (bottom). (d) The combined pulse and fitted hyperbolic secant pulse after $246 \mathrm{~m}$ length of propagation and (e) the output compressed pulse and fitted hyperbolic secant pulse at $300 \mathrm{~m}$. (f) The comparison of compression factor of the simulation and the theoretical self-similar pulse compressions during the final $54 \mathrm{~m}$ nonlinear fiber.

\section{Conclusion}

We illustrated the simultaneous combination and nearly self-similar pulse compression of input five chirped RC pulses separated both in temporal (cosine wave period) and spectral $(12.5 \mathrm{GHz})$ domains during the propagation in the $300 \mathrm{~m}$ exponentially dispersion decreasing nonlinear fiber. During the propagation, the combination length was found to be $246 \mathrm{~m}$ in which the FWHM pulse width decreased from $1 \mathrm{ps}$ to $0.206 \mathrm{ps}$ with a compression factor of 4.85. For the final compressed pulse, the FWHM was reduced from 1 ps to 78.17 fs corresponding to a compression factor of 12.8. The peak power was 27.63 times as compared to any peak of the initial $5 \mathrm{RC}$ pulses and the energy was $50 \%$ of total input light signal energy. The proposed compact passive optical fiber device can be used as an efficient multi-pulses combiner and compressor to generate high-energy ultrashort light pulses.

\section{Acknowledgment}

This work was supported by Shenzhen Technology and Innovation Council (Project GJHZ20180411185015272), Youth Science and Technology Innovation Talent of Guangdong Province (Project 2019TQ05X227) and research startup fund. K. Nakkeeran likes to acknowledge the Binks Trust Fund for this collaborative research project work.

\section{Reference}

[1] N. Picqué and T. W. Hänsch, "Frequency comb spectroscopy," Nat. Photonics 13, 146-157 (2019).

[2] M. Andreana, T. Le, W. Drexler, and A. Unterhuber, "Ultrashort pulse Kagome hollow-core photonic crystal fiber delivery for nonlinear optical imaging," Opt. Lett. 44, 1588 (2019).

[3] Q. Li, K. Senthilnathan, K. Nakkeeran, and P. K. A. Wai, "Nearly chirp- and pedestal-free pulse compression in nonlinear fiber Bragg gratings," J. Opt. Soc. Am. B 26, 432 (2009).

[4] Q. Li, Z. Jian, W. Lu, K. Nakkeeran, K. Senthilnathan, and P. K. A. Wai, "Combination and Compression of Multiple Optical Pulses in Nonlinear Fibers with the Exponentially Decreasing Dispersion," IEEE J. Quantum Electron. 54, 1-10 (2018).

[5] J. Huang, Q. Li, Z. Jian, P. K. A. Wai, and K. Nakkeeran, "Combination and Compression of Multiple Pulses with Same or Different Wavelengths,” IEEE J. Lightwave Technol., 38, 6932 - 6938 (2020). 